\title{
Fear of COVID-19: Psychological distress and coping among university students in Ghana
}

\author{
Mabel Oti-Boadi ${ }^{1}$ (D) $\cdot$ Esther Malm ${ }^{2} \cdot$ Nutifafa Eugene Yaw Dey $^{1}$ (D) Stephen Oppong ${ }^{1}$ \\ Accepted: 26 August 2021 / Published online: 5 September 2021 \\ (C) The Author(s), under exclusive licence to Springer Science+Business Media, LLC, part of Springer Nature 2021
}

\begin{abstract}
The advent of the coronavirus pandemic has impacted the psychological well-being of many people. This study examined the relationship between fear of COVID-19, psychological distress (depression, anxiety, and stress) and coping strategies adopted by undergraduate students in Ghana. A sample of 209 students were recruited to complete online surveys on fear of COVID-19, psychological distress and coping strategies between June and July 2020. Students scored between normal to mild levels of psychological distress but above average scores on fear of coronavirus $(M=19.45, S D=6.04)$. Fear of COVID-19 was positively related to psychological distress. Only maladaptive coping was found to be significantly and positively associated with fear of COVID-19. However post-hoc analysis of the components of coping strategies revealed that denial $(\beta=.17, p=$ $.028)$, venting ( $\beta=.18, p=.036)$ and humour $(\beta=-.18, p=.023$; an adaptive coping strategy) were associated with fear of COVID-19. Finally, both adaptive coping and maladaptive coping strategies had a mediating effect on fear of COVID-19 and psychological distress. These findings emphasize the need to design and optimize institutional interventions that will assess psychological distress and fear of COVID-19 levels during this pandemic and provide psychotherapeutic support for students as they return to school.
\end{abstract}

Keywords Fear of COVID-19 · Psychological distress $\cdot$ Coping $\cdot$ Undergraduates $\cdot$ Ghana

\section{Introduction}

The emergence of Coronavirus disease (COVID-19) has been associated with psychological distress in the general population globally (Rodríguez-Rey et al., 2020), as well as among students (Cao et al., 2020). The disease is a respiratory infection by a new virus called 2019-nCoV which the World Health Organization (WHO) officially named "2019 coronavirus disease" and is now popularly known across the world as COVID-19 (WHO, 2020a). This virus and its infections began in the Chinese city of Wuhan in 2019 and has since spread rapidly to every part of the world. As of November 23, 2020, the official statistics from the World Health Organization since December 2019 indicates that 218 countries/territories have had confirmed cases of the diseases. This translates to

Mabel Oti-Boadi

moti-boadi@ug.edu.gh; sweethour2014@gmail.com

1 Department of Psychology, University of Ghana, Legon, Accra, Ghana

2 Department of Psychology, Murray State University, Murray, KY, USA over 58 million people who have ever contracted the disease, and approximately $1,400,000$ people who have unfortunately lost their lives (WHO, 2020b). In Ghana, confirmed cases of COVID-19 have reached 50, 941 as of November 24, 2020, with 323 confirmed deaths (Ghana Health Service, 2020).

Due to the nature of the virus and how it spreads, many countries have instituted strict quarantine and isolation measures amid closure of organizations and schools. Many schools and colleges have had to resort to online learning, with uncertainty about reopening or appropriate protocols to implement. This uncertainty and rapid shift in learning formats have been found to create distress among students (Cao et al., 2020). Many students experience psychological distress as a result of their inability to afford internet data for online class platforms usage, have strong internet reception in their area, and smooth transition to online learning (Kapasia et al., 2020). Whereas the physical impact of COVID-19 has been broadly explored (Shaukat et al., 2020), emerging research is indicating the psychological impact across different age groups could be grave and should be urgently studied (Holmes et al., 2020).

The psychological impact of COVID-19 has been found to be exhibited in distress related symptoms including stress, depression and anxiety (Serafini et al., 2020). Social isolation and other restrictive measures have also been associated with 
fears of the unknown and feelings of uncertainty which also results in pervasive anxiety ( $\mathrm{Li}$ et al., 2020). It has become evident that when social interactions are curtailed, as experienced during this pandemic, they result in psychological distress (de Lima et al., 2020). Psychological distress has therefore been found to be vital in assessing the mental state of students, as distress (regardless of source) has often been found to precede many psychological disorders which in turn negatively affects students' learning and academic performances (Eisenbeck et al., 2019).

The lack of certainty about COVID-19 and its future impact has also been found to be associated with feelings of unpredictability of events, and fears (Satici et al., 2020). For example, the fear of contracting an infectious disease like COVID-19 has been found to play a huge role in the experience of psychological distress (Labrague \& De los Santos, 2020). The fear of the unknown associated with pandemics has been attributed to the uncertainty surrounding the transmission of the infection and the susceptibility of various populations to infection and the disease (Satici et al., 2020). Extant literature shows that people exposed to risk of any infection may develop fears of transmission, fear of their health, and fear of infecting others (Ahorsu et al., 2020).

\section{Coping Strategies and COVID-19 Pandemic}

When problems, uncertainties and fear occur, however, people develop different strategies for handling them. These strategies originate from thoughts, feelings and actions that individuals use in dealing with stressful situations and are broadly known as coping strategies (Lazarus, 1999). Use or reuse of coping strategies can depend on a variety of factors including past experiences, speed of relief needed, availability and value placed on the strategy, impact and severity of the stressful or traumatic situation (Carver, 1997; Wang et al., 2020). Coping strategies can be grouped in many ways. One way is focusing on coping strategies that generally lead to adaptive versus maladaptive outcomes (e.g., Kilbane et al., 2020). Examples of adaptive coping strategies include positive reframing, use of social support, religious coping, acceptance and humor while maladaptive forms of coping include denial, blaming, social withdrawal, substance use and disengagement, all of which are used to manage the difficulties associated with emergency situations (Carver, 1997; Skeffington et al., 2017). Generally, experiences of psychological distress have been found to be positively associated with use of maladaptive coping styles (Umucu \& Lee, 2020), whilst use of adaptive coping styles tend to be associated with positive influences on psychological well-being (Wang et al., 2020). Coping strategies have also been found to be significant in managing the psychological distress associated with traumatic events such as pandemics (Khalid et al., 2016).
In reference to the COVID-19 pandemic, two recently published studies have reported the important mediational role of coping strategies in handling COVID-19 related stress. In one study, adaptive coping strategies rather than maladaptive were found to buffer the relationship between COVID-19-related stressful experiences and acute stress disorder among Chinese college students (Ye et al., 2020). In the other study conducted in the United Kingdom, Rettie and Daniels (2020) found that only maladaptive coping strategies significantly mediated the relationship between intolerance of uncertainty and mental health difficulties examined (depression, generalized anxiety and health anxiety). Although these findings collectively offer useful information about the relevance of coping strategies associated with distress in this pandemic era, there are currently mixed findings and context specific factors that may be influencing published findings so far, calling for more research into the utilization of coping strategies during COVID-19 and also across cultures.

\section{Psychosocial Impact of COVID-19 across Cultures/Countries}

Most studies on COVID-19 and health and psychosocial outcomes on psychological well-being have emerged from China (e.g., Cao et al., 2020; Wang et al., 2020) and other Western countries (e.g., Bäuerle et al., 2020; Rodríguez-Rey et al., 2020). In the study by Rodríguez-Rey et al. (2020) in Spain, authors found that among the 3055 participants sampled, many Spaniards considered the health impact of COVID- 19 as fairly severe with about $36 \%$ of the participants reporting moderate to severe psychological impact, $25 \%$ showed mild to severe levels of anxiety, $41 \%$ reported depressive symptoms, and $41 \%$ felt stressed. In another study that focused on students from China, Cao et al. (2020) found that of the 7143 college students, less than $4 \%$ experienced severe or moderate anxiety, while about $21 \%$ experienced mild anxiety. They further found significant positive associations between economic, daily life, delays in academic activities and anxiety symptoms due to COVID-19.

\section{The Present Study}

To our knowledge, no study has yet been conducted in Ghana among university students to examine how fear of COVID-19 contributes to psychological distress. The current study also seeks to determine the role of coping in the experience of psychological distress. Identifying and understanding the psychological processes associated with the presence of COVID19 among students will significantly contribute to understanding the kinds of interventions to be considered by university administrators and policy makers on how to effectively manage and support students. It was therefore hypothesized that fear of COVID-19 will be significantly and positively 
associated with psychological distress (depression, anxiety and stress). Secondly, adaptive coping strategies will be significantly and negatively associated with fear of COVID-19 while maladaptive coping will be positively associated with fear of COVID-19. Lastly, we hypothesized that both adaptive and maladaptive coping strategies will fully mediate the relationship between fear of COVID-19 and depression, anxiety and stress in expected directions.

\section{Methods}

\section{Research Design}

A correlational design was adopted for this study among university undergraduate students with data collection exclusively conducted online using a survey designed in Google Forms. Data was collected between June and July 2020. Measures used in the study comprised demographic fact sheets, and standardized questionnaires. Both purposive and the snowball sampling methods were used to invite participants. Participants could share questionnaires with friends on social media platforms.

\section{Participants}

Participants were students from the University of Ghana. A sample of 214 participants were purposively recruited using a strategy of active link sharing on University of Ghana students' social media group platform: WhatsApp. Inclusion criteria to participate in the study were (i) be a Ghanaian attending the University of Ghana, (ii) aged 18 years or older and (iii) being able to understand English. Participants voluntarily responded to informed consent before responding to a set of measures which assessed coping strategies, fear of COVID-19, psychological distress, as well as demographic questions. Participants accessed the survey by clicking on the shared link. The survey took approximately 20-30 min. The final sample after excluding individuals with $50 \%$ incomplete or missing responses were 209 students. Adequacy of the sample size was determined by G*Power 3.1.9.2 (Faul et al., 2007) using the option of a priori power analysis based on Cohen's (1992) acceptable power of .80 , an alpha of 0.05 , a medium effect size of 0.15 and number of predictors set at 14 . This power analysis indicated a minimum of 135 participants as sufficient to detect medium effect. The demographic characteristics of participants are as follows: majority identified as male $(64.1 \%)$, were in third year of their undergraduate education (36.8\%) and identified as Christians (93.8\%). Participant ages ranged from 18 to $28(M=21.54, S D=$ 2.04).

\section{Measures}

The online survey, in addition to the main standardized questionnaires, contained a demographic sheet designed to capture certain biodata from students. The demographic information thus collected data on the age, sex, level in school (educational year) and religious affiliation.

The Fear of COVID-19 Scale (Ahorsu et al., 2020) The Fear of COVID-19 Scale was used to examine students' fear about COVID-19. This 7-item unidimensional scale was originally used with a sample of nurses using a 5-point Likert scale which ranged from 1 (strongly disagree) to 5 (strongly agree). The composite score ranged from 7 to 35 , with a higher score indicating greater fear of COVID-19. Previous research reported excellent predictive validity and reliability $(\alpha=$ 0.88) of the scale (Bakioğlu et al., 2020). The Cronbach's $\alpha$ of the scale in the present study was 0.87 .

Depression, Anxiety, and Stress Scales (DASS-21; Lovibond \& Lovibond, 1995) The DASS-21 is a 21-item self-report instrument for mental health assessment consisting of three 7-item subscales: depression (items 3, 5, 10, 13, 16, 17, and 21), anxiety (items 2, 4, 7, 9, 15, 19, and 20), and stress (1, 6, 8, 11, 12, 14, and 18). Participants indicate on a 4-point Likert scale ranging from 0 (Did not apply to me at all) to 3 (Applied to me very much, or most of the time) the degree in which a symptom had been present over the past week. Scores for each subscale were computed by summing the item responses (Lovibond \& Lovibond, 1995; Zanon et al., 2020). The use of DASS-21 for COVID-19 related distress was scored using similar COVID-19 research findings, therefore the summed scores were doubled to make them comparable to similar COVID-19 research (Wang et al., 2020). The subscales scores can be allocated on one of 5 levels of severity, as described in Wang et al. (2020). Thus, for depression, scores range from normal (0-9), mild (10-12), moderate (13-20), severe (21-27), to extremely severe (28-42). For anxiety, scores ranged from normal (0-6), mild (7-9), moderate $(10-14)$, severe (15-19), to extremely severe (20-42). Finally, for stress levels, scores ranged from normal $(0-10)$, mild (11-18), moderate (19-26), severe (27-34), to extremely severe (35-42). The psychometric properties of DASS-21 have remained moderate to strong over time and have been used across many cultures (Zanon et al., 2020). Reliability scores from the research by Park et al. (2020) on depression, anxiety and stress were $.93, .84$ and .88 , respectively. The Cronbach's $\alpha$ of each subscale for the present study were $.88, .83$ and .86 in similar order.

The Brief Coping Inventory (Carver, 1997) The Brief Cope Inventory is a 28-item questionnaire developed from the original version of the Cope Inventory which was made up of 60 
items. Thus, this inventory offers a faster and more convenient way of assessing coping strategies. This scale assesses 14 different coping reactions (factors) which could be generally adaptive or maladaptive strategies. The 28 items on the Brief Cope Inventory measures 14 factors, covered by 2 items each. They include Self-distraction (1 and 19); Active coping (2 and 7); Denial (3 and 8); Substance use (4 and 11); Use of emotional support (5 and 15); Use of instrumental support (10 and 23); Behavioural disengagement (6 and 16); Venting (9 and 21); Positive reframing (12 and 17); Planning (14 and 25); Humour (18 and 28); Acceptance (20 and 24); Religion (22 and 27); and Self-blame (13 and 26). The response format ranges from 1 ("I haven't been doing this at all") to 4 ("I've been doing this a lot"). Higher scores reflect better coping strategies while lower scores mean poor coping strategies, with the total highest score of 112 and the lowest possible score being 28. García et al. (2018) reported internal consistencies ranging from $\alpha=.53$ to $\alpha=.82$. In the present study, Cronbach's $\alpha$ of each subscale ranged from .47-.86. Table 4 in appendix outlines the reliability score for each subscale.

\section{Ethical Considerations}

This study was approved by the ethics committee, Department of Psychology, University of Ghana (DREC/016/19-20). Participation was voluntary and anonymized. Participants were informed about the purpose of the study, voluntarily consented to participation before receiving access to the questionnaires. Participants were also informed of their opportunity to withdraw from the research at any time.

\section{Data Analysis}

All statistical analyses were conducted with IBM SPSS Statistics version 23 with significance set at $p \leq 0.05$. Frequencies and percentages were performed for the demographic variables. Means, standard deviations, reliability values (i.e., Cronbach alphas) and normality checks were calculated for all the main study variables. A summary of the descriptive analyses is reported in Table 1 . The Pearson product-moment correlation was used to analyze the strength and direction between all variables. A standard multiple regression was used to examine the relationship between coping strategies and the fear of COVID-19.

Lastly, PROCESS macro for SPSS, version 3.4 (Model 4; Hayes \& Rockwood, 2020) was used to test the mediating effects of adaptive coping (M1) and maladaptive coping (M2) on the relationship between fear of COVID-19 (X) and depression (Y1), anxiety (Y2), and stress (Y3). Model 4 can be used for both the traditional single mediation analyses, as well as mediation of two related variables (Hayes \& Rockwood, 2020). As such, three separate mediation analyses were conducted with adaptive and maladaptive coping entered together as mediators for three outcomes. Following Hayes (2009) recommendation on bootstrapping, we set the estimator at 5000 bootstrap samples to ensure a more valid and robust testing procedure for mediating effects in this study. Bootstrapped $95 \%$ bias corrected confidence intervals (CI) not containing zero were considered as significant values, and in determining the presence of mediation (Preacher \& Hayes, 2008).

\section{Results}

\section{Descriptive and Correlation Analyses}

A close examination of the mean scores revealed that the most commonly used coping strategies by students was religion $(M$ $=5.87, S D=1.81)$, followed by planning $(M=5.77, S D=$ $1.75)$ and self-distraction $(M=5.74, S D=1.85)$. The least used on the other hand were substance use $(M=2.11, S D=$ $.31)$, behavioural disengagement $(M=3.48, S D=1.44)$, and denial $(M=3.86, S D=1.66)$. See these results in Table 4 [Appendix]. Correlation analyses (in Table 2) indicated that Fear of COVID-19 was significantly and positively associated with depression symptoms $(r=.37, p<.001)$, anxiety $(r=$ $.42, p<.001)$ and stress $(r=.42, p<.001)$ as expected. Adaptive coping was only positively related to anxiety $(r=$ $.15, p=.030)$ and Stress $(r=.14, p=.037)$. Maladaptive
Table 1 Descriptive and preliminary results of main study variables

\begin{tabular}{lllllll}
\hline & Mean & $S D$ & $\alpha$ & Skewness & Kurtosis & $\begin{array}{c}\text { Min- } \\
\text { Max }\end{array}$ \\
\hline 1. Depression & 5.18 & 4.56 & .88 & 1.04 & .91 & $0-21$ \\
2. Anxiety & 4.72 & 4.23 & .83 & 1.16 & 1.02 & $0-19$ \\
3. Stress & 5.78 & 4.43 & .86 & .67 & -.11 & $0-19$ \\
4. Fear of COVID-19 & 19.45 & 6.04 & .86 & .078 & -.276 & $7-34$ \\
5. Adaptive Coping & 42.25 & 9.20 & .88 & -.328 & .359 & $16-64$ \\
6. Maladaptive Coping & 24.07 & 6.00 & .78 & .225 & -.150 & $12-42$ \\
\hline
\end{tabular}

Note. SD Standard deviation; $\alpha=$ Cronbach's Alpha 
Table 2 Results of zero-order correlation analysis of study variables (n = 209)

\begin{tabular}{lllllll}
\hline & 1 & 2 & 3 & 4 & 5 & 6 \\
\hline 1. Depression & - & & & & \\
2. Anxiety & $.766^{* *}$ & - & & & \\
3. Stress & $.818^{* *}$ & $.816^{* *}$ & - & & \\
4. Fear of COVID-19 & $.367^{* *}$ & $.421^{* *}$ & $.415^{* *}$ & - & \\
5. Adaptive Coping & .063 & $.151^{* *}$ & $.144^{*}$ & .129 & - & \\
6. Maladaptive Coping & $.557^{* *}$ & $.541^{* * *}$ & $.558^{* *}$ & $.330^{* *}$ & $.514^{* *}$ & - \\
\hline
\end{tabular}

Note. $* * p \leq .01 ; * p \leq .05$

coping was positively related to depression $(r=.56, p<.001)$, anxiety $(r=.54, p<.001)$, stress $(r=.56, p<.001)$ and fear of COVID-19 $(r=.33, p<.001)$.

\section{Multiple Regression Analyses}

The significant independent predictor of fear of COVID-19 (FC) was maladaptive coping. The total model, which was significant, $(F(2,206)=12.84, p>.001)$ accounted for $11 \%$ of the variance in FC. Out of curiosity a post hoc multivariate analysis in multiple regression was conducted to examine which specific coping strategies were associated with fear of COVID-19. The overall model after entering the list of 14 predictors crudely into the model was statistically significant $\left(R^{2}=.20, F(14,194)=3.46, p\right.$ $<.001)$ with denial $(\beta=.17, p=.028)$, venting $(\beta=.18$, $p=.036)$ and humour $(\beta=-.18, p=.023)$ as three key coping strategies contributing significantly to the model. Humor, the only significant adaptive coping made the strongest unique contribution to the variance of the model and was negatively associated with fear of COVID-19 (See Table 4 in Appendix).

\section{Mediation Analysis}

The results for all three parallel mediational analyses (total effect, direct effect, and indirect effect) are presented below and on Table 3.

\section{Total Effect of Fear of COVID-19 on Depression, Anxiety, and Stress}

The total effect of fear of COVID-19 on depression $(t(207)=$ $5.67, B=.28, p<.001)$, anxiety $(t(207)=6.68, B=.30, p$ $<.001)$ and stress $(t(207)=6.57, B=.30, p<.001)$ while not controlling for the mediators were statistically significant.

\section{Direct Effect of Fear of COVID-19 on Depression, Anxiety, and Stress}

Fear of COVID-19 in this model also significantly predicted depression $(t(205)=3.38, B=.15, p<.001)$, anxiety $(t(205)=4.54, B=.19, p<.001)$ and stress $(t(205)=4.36$, $B=.18, p<.001$ ), while controlling for the mediators. This result further confirmed our hypothesis that fear of COVID-19 will be positively related to depression, anxiety, and stress.

Also significant in the model were the associations between adaptive coping on depression $(t(205)=-4.70, B=$ $-.15, p<.001)$, anxiety $(t(205)=-2.47, B=-.07, p=$ $.014)$ and stress $(t(205)=-2.84, B=-.09, p=.005)$. Similarly, the association between maladaptive coping and depression $(t(205)=9.82, B=.49, p=.001)$, anxiety $(t(205)=7.93, B=.38, p<.001)$ and stress $(t(205)=$ $8.52, B=.42, p<.001)$ were also significant.

\section{Indirect Effects of Fear of COVID-19 on Depression, Anxiety, and Stress}

The mediation analysis is visually presented in Figs. 1, 2 and 3, collectively showing fear of COVID-19 as the predictor variable, adaptive and maladaptive coping as the mediator variables, as well as depression, anxiety, and stress as the outcome variables with unstandardized coefficients on each regression pathway namely $\mathrm{a}, \mathrm{b}, \mathrm{c}$, and c'. The visual representation indicates that both adaptive coping and maladaptive coping strategies mediated the relationship between fear of COVID-19 and depression, anxiety, and stress. In more simple terms, students who exhibited higher fear of COVID-19 used adaptive coping to reduce their levels of depression $\left(t(205)=-4.70, \mathrm{~b}^{1}=-.15, p<.001\right)$, anxiety $\left(t(205)=-2.47, \mathrm{~b}^{1}=-.07, p=.014\right)$, and stress $\left(t(205)=-2.84, \mathrm{~b}^{1}=-.09, p=.005\right)$. Also, students with higher fear of COVID-19 who used maladaptive coping strategies experienced increased levels of depression $(t(205)=9.82$, $\left.\mathrm{b}^{2}=.49, p=.001\right)$, anxiety $\left(t(205)=7.93, \mathrm{~b}^{2}=.38, p<.001\right)$, and stress $\left(t(205)=8.52, \mathrm{~b}^{2}=.42, p<.001\right)$.

Further results in Table 3 show that the bootstrap estimation procedure (with a defined bootstrap sample of 5000) supports these significant indirect effects. Specifically, fear of COVID-19 had a statistically significant indirect effect through adaptive coping strategy on Depression $\left(\mathrm{a}^{1} \mathrm{~b}^{1}=\right.$ $-.03 ; 95 \%$ CI: $-.07,-.001)$, Anxiety $\left(\mathrm{a}^{1} \mathrm{~b}^{1}=-.01 ; 95 \%\right.$ CI: $-.04,-.0001)$ and finally Stress $\left(\mathrm{a}^{1} \mathrm{~b}^{1}=-.02 ; 95 \%\right.$ CI: -.05 , $-.0002)$ as the confidence interval completely excluded zero. Through maladaptive coping, fear of COVID-19 also significantly influenced Depression $\left(\mathrm{a}^{2} \mathrm{~b}^{2}=.04 ; 95 \%\right.$ CI: .02, .05), Anxiety $\left(\mathrm{a}^{2} \mathrm{~b}^{2}=.12 ; 95 \%\right.$ CI: .07, .19) and Stress $\left(\mathrm{a}^{2} \mathrm{~b}^{2}=.14\right.$; $95 \%$ CI: .08, .21). In sum, both adaptive coping and maladaptive coping mediated the relationship between fear of COVID19 and depression, anxiety, and stress among Ghanaian University students. 
Table 3 Results of regression and mediation pathways of adaptive coping, maladaptive coping, fear of Covid-19 and the three indexes of psychological distress

\begin{tabular}{|c|c|c|c|c|c|c|c|c|}
\hline \multirow[t]{2}{*}{ Predictors } & \multicolumn{2}{|l|}{ Depression } & \multicolumn{2}{|l|}{ Anxiety } & \multicolumn{2}{|l|}{ Stress } & $\begin{array}{l}\text { Adaptive Coping } \\
\text { (AC) }\end{array}$ & $\begin{array}{l}\text { Maladaptive } \\
\text { Coping (MC) }\end{array}$ \\
\hline & $B(\mathrm{SE} B)$ & $p$ & $B(\mathrm{SE} B)$ & $p$ & $B(\mathrm{SE} B)$ & $p$ & $B(\mathrm{SE} B) \quad p$ & $B(\mathrm{SE} B)$ \\
\hline Total effect & \multicolumn{2}{|c|}{$\begin{array}{l}R^{2}=.14, F(1,207)= \\
\quad 32.17, p<.001\end{array}$} & \multicolumn{2}{|c|}{$\begin{aligned} R^{2} & =.18, F(1,207)=44.71 \\
& p<.001\end{aligned}$} & \multicolumn{2}{|c|}{$\begin{aligned} R^{2} & =.17, F(1,207)=43.10, \\
& p<.001\end{aligned}$} & $\begin{array}{c}R^{2}=.12, F(1,207)= \\
3.50, p=.063\end{array}$ & $\begin{aligned} R^{2} & =.11, F(1,207) \\
& =25.23, p<.001\end{aligned}$ \\
\hline Fear of COVID-19 (FC) & $.28,(.05)$ & .000 & $.30(.04)$ & .000 & $.30(.05)$ & .000 & $.20(.11)$ & $.33(.07)$ \\
\hline Direct Effect & \multicolumn{2}{|c|}{$\begin{array}{c}R^{2}=.41, F(3,205)= \\
47.80, p<.001\end{array}$} & \multicolumn{2}{|c|}{$\begin{array}{l}R^{2}=.38, F(3,205)=41.50, \\
\quad p<.001\end{array}$} & \multicolumn{2}{|c|}{$\begin{aligned} R^{2} & =.40, F(3,205)=44.61, \\
& p<.001\end{aligned}$} & & \\
\hline $\mathrm{FC}$ & $.15(.04)$ & .001 & $.19(.04)$ & .000 & $.18(.04)$ & .000 & & \\
\hline $\mathrm{AC}$ & $-.15(.03)$ & .000 & $-.07(.03)$ & .014 & $-.09(.03)$ & .005 & & \\
\hline $\mathrm{MC}$ & $.49(.05)$ & .001 & $.38(.05)$ & .000 & $.42(.05)$ & .000 & & \\
\hline Indirect effect $95 \% \mathrm{CI}$ & & BLL, BUL & & BLL, BUL & & BLL, BUL & & \\
\hline $\mathrm{FC} \rightarrow \mathrm{AC}$ & $-.03(.02)$ & $-.07,-.001$ & $-.01(.01)$ & $-.04,-.0001$ & $-.02(.01)$ & $-.05,-.0002$ & & \\
\hline $\mathrm{FC} \rightarrow \mathrm{MC}$ & $.04(.01)$ & $.02, .05$ & $.12(.03)$ & $.07, .19$ & $.14(.03)$ & $.08, .21$ & & \\
\hline
\end{tabular}

$B$ unstandardized coefficient, $C I$ confidence interval, $S E$ standard error. Bootstrapped samples $=5000$. $B L L$ Bootstrapped Lower Limit, $B U L$ Bootstrapped Upper Limit

\section{Discussion}

The current study sought to determine the psychological distress experienced by students during the outbreak of the COVID-19 pandemic and the strategies adopted in coping. Results from this study show that fear of COVID-19 was positively associated with psychological distress as well as maladaptive coping strategies. Both adaptive and maladaptive coping mediated the relationship between fear of COVID-19 and psychological distress - depression, anxiety, and stress.

Elevated fear of COVID-19 was associated with increased depression, anxiety and stress levels of students in the present study, and this is in congruence with emerging research from other populations during this pandemic (Bakioğlu et al., 2020; Labrague \& De los Santos, 2020; Satici et al., 2020). These significant patterns are probably due to the novelty of the virus, the avid consumption of negative news, lowering levels of sense of control, the increased sense of uncertainty, distorted thinking patterns, and associated national restrictions which put tremendous strain on the well-being of students in this study (Fofana et al., 2020).

In addition to these psychosocial explanations, there may be some interacting biological basis for these relationships. Plausibly, the increased levels of fear of COVID-19 activated the hypothalamic-pituitary-adrenal axis (HPA), triggering the release of stress hormones including cortisol which in turn raised students' vulnerability (e.g., altered appraisals) towards the developments of depression, anxiety, and stress (Bao \& Swaab, 2019). However, it is worth noting that, based on average scores, participants reported normal to mild levels of depression, anxiety, and stress. Also, the mean score ( $M=$ $19.45, S D=6.04$ ) of fear of COVID-19 put our participants at a high comparable rate similar to those found in other studies amongst students (Bakioğlu et al., 2020) and frontline nurses (Labrague \& De los Santos, 2020).

Findings about the relationship between maladaptive coping and fear of COVID-19 corroborates with previous studies which have shown the importance of coping in life threatening situations (Taha et al., 2014) and the use of coping strategies to handle fear of any situation (Oplatka \& Iglan, 2020). However, this study only found maladaptive coping to be associated with fear of COVID-19 among students. This implies that students in this study only used maladaptive coping to deal with their fears of COVID-19 during the data collection period when they were in isolation, having lectures and writing exams remotely.

It is also possible that students' choice of coping strategies to deal with fear of COVID-19 was inspired by their feeling of loss of control. Evidence from literature shows that people's sense of control was threatened during the early stages of the pandemic due to reasons including uncertainty, incomplete information about the virus, involuntary changes in behaviours, the restrictions like social distancing and isolation, and apparent financial difficulties (Wnuk et al., 2020). Low sense of control is related to maladaptive coping (Dijkstra \& Homan, 2016) and in the case of our participants, this sequence of events may have influenced the adoption of more maladaptive coping than adaptive coping strategies against fear of COVID-19.

To further understand which specific coping strategies related to fear of COVID-19, we performed post hoc analysis (See Table 4 in Appendix for results on specific coping strategies). Together, maladaptive coping strategies such as denial and venting were significantly related to fear of COVID-19 
Fig. 1 Observed model for the mediation of fear of COVID-19 on Depression through adaptive and maladaptive coping strategies; $a$ = IV to mediator path, $\mathrm{b}=$ mediator to DV path, $\mathrm{c}$ $=$ total effect path, and c' = direct effect path controlling for both mediators. $* p \leq .05$. Long dashes represent non-significant paths while solid lines represent significant paths

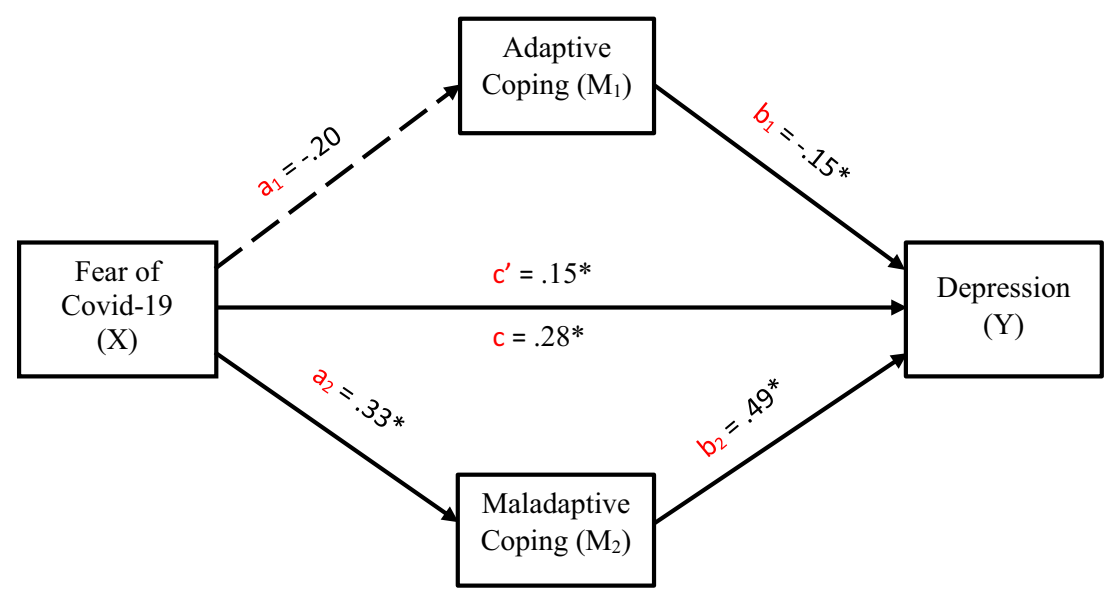

than humour, the only (strongest individual predictor and) significant adaptive coping. Our results suggest that the use of denial (the refusal to accept statements or truths) and venting (the act of discharging negative emotions) as a coping strategy increased the fears of COVID-19 among students. These findings are consistent with previous studies that have shown that denial is positively related to the perceived threat of developing cardiovascular disease (Thompson \& Ting, 2012) and emotional venting is used to manage emotional distress in crisis situations (Jin et al., 2016). It also agrees with findings of a recent study demonstrating that denial and venting were related to COVID-19 stress among people with disability (Umucu \& Lee, 2020). Denial in this pandemic has been noted to manifest in the tendency to hold strong false beliefs and conspiracy theories, to display confirmation bias, and to reject science (Miller, 2020). Indeed, emerging literature suggests that those who held varying views and theories about the virus, its source, spread and motive, were less likely to comply with public health safety measures (Romer \& Jamieson, 2020). Our finding about the use of denial and venting therefore highlights the need to provide comprehensive public health education about COVID-19 and its related complications.
In terms of adaptive coping, only humour (the experience of or ability to elicit laughter or amusement) was found to significantly reduce fear of COVID-19, resonating with past literature that has found humour as a valuable coping strategy in reducing stress and promoting emotional well-being (Crawford \& Caltabiano, 2011; Maxwell, 2003). This finding is especially interesting and reflective of the early months of the pandemic where COVID-19 memes, short comic videos and dark humor were highly dispersed on social media and avidly consumed by the youth (Bischetti et al., 2020). An example of one of these viral coronavirus memes (which originated from Ghana) is the coronavirus electronic music dancing pallbearers (Sullivan, 2020). It is worth mentioning that the commonly used coping strategy among participants was religion. Religion plays a protective role in the lives of Africans including Ghanaians who have been described as "notoriously religious" (Mbiti, 1969). Although this finding emphasizes participants' strong belief systems and its general usage, it further highlights the need to establish collaborations with religious institutions to aid the campaign against the spread of the virus.

Finally, both adaptive and maladaptive coping strategies significantly mediated the relationship between fear of COVID-19 and psychological outcomes. In order words,
Fig. 2 Observed model for the mediation of fear of COVID-19 on Anxiety through adaptive and maladaptive coping strategies; $a$ $=\mathrm{IV}$ to mediator path, $\mathrm{b}=$ mediator to DV path, $\mathrm{c}=$ total effect path and c' $=$ direct effect path controlling for both mediators. $* p \leq .05$. Long dashes represent non-significant paths while solid lines represent significant paths

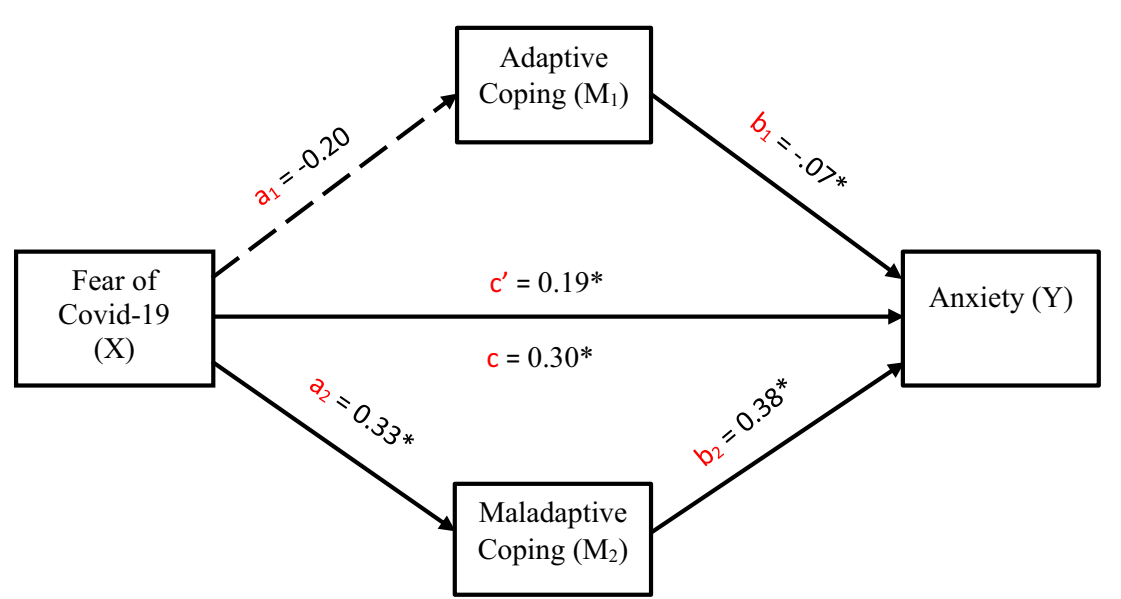


Fig. 3 Observed model for the mediation of fear of COVID-19 on Stress through adaptive and maladaptive coping strategies; $a$ $=\mathrm{IV}$ to mediator path, $\mathrm{b}=$ mediator to DV path, $\mathrm{c}=$ total effect path and c' $=$ direct effect path controlling for both mediators. $* p \leq .05$. Long dashes represent non-significant paths while solid lines represent significant paths

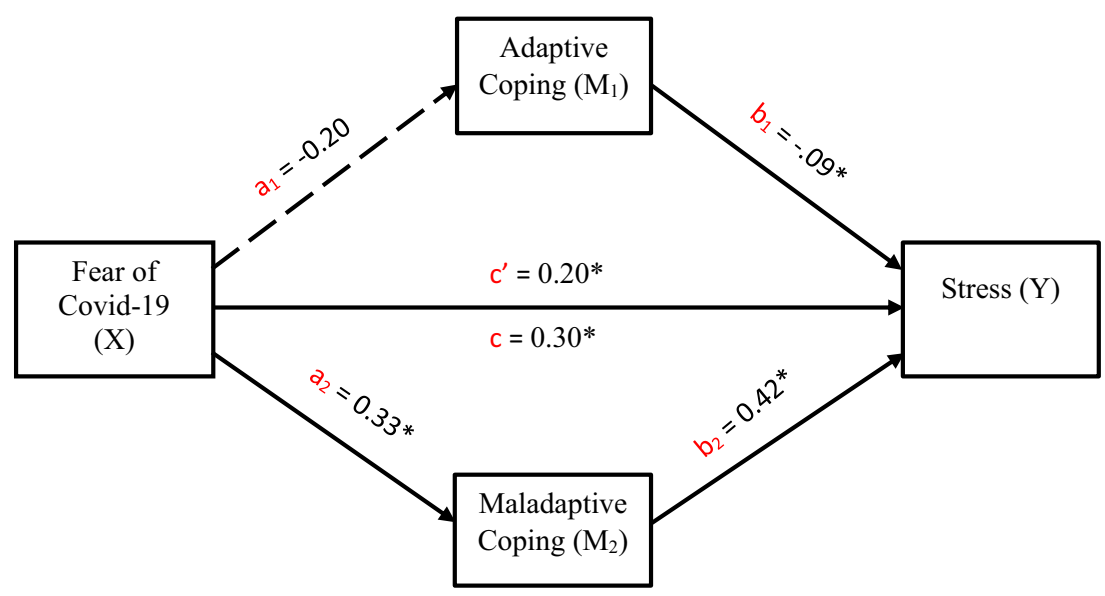

students' increased experience of fear of COVID-19 either propelled the use of adaptive coping to reduce their depression, anxiety, and stress levels or the use of maladaptive coping strategies which sadly increased their levels of depression, anxiety, and stress. These findings collectively correspond with recent literature indicating that adaptive and maladaptive coping strategies mediate the relationship between coronavirus related predictors and psychological outcomes (Rettie \& Daniels, 2020; Ye et al., 2020). As already established, these parallel mediation findings can be explained in light of the experience of loss of control, which weakened the use of adaptive coping and reinforced the use of maladaptive coping. It is also likely that participants' increased use of maladaptive coping and decreased use of adaptive coping to deal with the pandemic was due to poor appraisal of their fear responses (Folkman \& Lazarus, 1980).

Moreover, individuals with greater levels of fear are less likely to use adaptive coping; they may instead choose maladaptive coping strategies like avoidance and denial that prevents them from positively dealing with the stressful event (Carver et al., 1989). Maladaptive coping increases the vulnerability to distress after an exposure to a traumatic event (Lalande \& Bonanno, 2011). Nevertheless, owing to the modifiable nature of coping strategies, its strong link in the subsequent development of psychological problems (Lee et al., 2013) and the never-ending impact of coronavirus, it becomes imperative to mechanistically incorporate coping strategies in the optimization of treatments for students by counsellors and psychologists on their return to school (Holmes et al., 2020).

\section{Implications for student's Mental Health}

The findings of the study have significant implications for student's mental health in terms of the development or optimization of interventions that seek to provide support and appropriate coping strategies in times of crisis like a pandemic. The evidence of fear of COVID-19 and its subsequent negative associations with psychological distress highlight the important role of increasing awareness for all health professionals and educational personnel including school counsellors and psychologists, in preparation towards reopening of schools and with the general psychological adjustment of students. Since research has shown that people with adequate and appropriate information about COVID-19 exhibit less psychological distress (e.g., Siebenhaar et al., 2020), training programmes should be organized over various social media and video conferencing platforms on issues regarding COVID-19, distress, academic achievement, and adaptive coping strategies in order to allay the fears students have or will have. Such interventions will also help them adapt to the present situation, improve their well-being, and ameliorate their risk of developing other psychological disorders in the future. Additionally, education campaigns should be launched by the university to further promote public health messages that avert the spread of the disease and inspire the public to take practical steps, such as reporting signs of illness to health professionals. There should also be a collaboration between government, health agencies, religious leaders and education institutions to provide a comprehensive crisis oriented psychological services to reduce psychological distress and prevent future mental health problems by encouraging students to use more adaptive coping strategies and less maladaptive ones.

\section{Strengths and Limitations}

One strength of this paper is its addition to literature specifically on the fear of COVID-19. Findings add evidence about the effects of the pandemic on the psychological health of students from a low-and-middle-income country. It also reinforces the few existing literature examining the direct and indirect contributions of coping strategies on psychological health during this pandemic. Nevertheless, there are some limitations worth noting. The correlational design utilized for this study makes it difficult to establish causation between the variables under investigation. Longitudinal studies are 
recommended in future research to strengthen and augment the understanding of how students utilize adaptive and maladaptive coping strategies in feared situations over time, and how that might also influence their psychological distress namely, anxiety, depression and stress. Further, although the sample size was deemed statistically adequate, the study was conducted among a cross-sectional sample of students recruited from only one university in Ghana, limiting randomization and representativeness of the sample. This means that care must be taken when generalizing the findings to a larger and more prominent student population in Ghana. It is therefore recommended that future studies should either be conducted on a larger and more representative sample of students or other populations to achieve adequate generalization. Another potential limitation was the use of self-report measures. It is possible that students may have under-or-over reported their experiences which are susceptible to socially desirable outcomes. In addition, although the online survey offered a convenient means of data collection especially during the period when the pandemic related lockdown and social distancing protocols were in full force, it may have led to a biased data due to sampling error. For instance, a participant could have shared the survey link with non-targeted groups who may have decided to participate in the survey by feigning characteristics consistent with the inclusion criteria thus, making it difficult to screen out their responses. Lastly, despite the dem- onstrated robustness of our analyses, we believe that some confounding may have occurred from not statistically controlling for potential covariates (e.g., students' demographic characteristics).

\section{Conclusion}

This study highlights the negative psychological effects of COVID-19 among Ghanaian university students. Participants exhibited high levels of psychological distress emanating from their fear of contracting COVID-19. There was also evidence of utilizing various coping strategies in dealing with their distress. However, maladaptive coping strategies emerged as the commonest coping strategies employed by students faced with a threatening health condition like COVID-19. Humor was the only significant adaptive coping strategy associated with lowering fear of COVID-19 in this sample. Finally, both adaptive and maladaptive coping strategies were found to mediate the relationship between fear of COVID-19. Findings from this study has the potential to help in the design and implementation of appropriate interventions to support students who have experienced distress during the pandemic to cope better. The current study also serves as a springboard for further studies regarding the psychological impact of COVID-19 in Ghana.

\section{Appendix}

Table 4 Descriptive analysis and Standard multiple regression analysis for coping strategies predicting Fear of Covid-19 $(\mathrm{n}=$ 209)

\begin{tabular}{llllllll}
\hline Model & $M(S D)$ & $B(\mathrm{SE} B)$ & $\beta$ & $t$ & Sig. & LB & UB \\
\hline Constant) & & $9.25(3.48)$ & & 2.66 & .008 & 2.39 & 16.12 \\
Self-distraction $(\alpha=.67)$ & $5.74(1.85)$ & $.51(.27)$ & .16 & 1.85 & .066 & -.03 & 1.04 \\
Substance use $(\alpha=.71)$ & $2.11(.31)$ & $1.70(1.37)$ & .09 & 1.24 & .216 & -1.00 & 4.41 \\
Behavioral disengagement $(\alpha=.59)$ & $3.48(1.44)$ & $.66(.34)$ & .16 & 1.92 & .056 & -.02 & 1.33 \\
Venting $(\alpha=.51)$ & $\mathbf{4 . 5 0}(\mathbf{1 . 6 6})$ & $\mathbf{. 6 4}(. \mathbf{3 0})$ &. $\mathbf{1 8}$ & $\mathbf{2 . 1 1}$ & $\mathbf{. 0 3 6}$ & $\mathbf{. 0 4}$ & $\mathbf{1 . 2 3}$ \\
Self-blame $(\alpha=.78)$ & $4.29(1.96)$ & $-.30(.26)$ & -.10 & -1.13 & .261 & -.82 & .22 \\
Denial $(\alpha=.64)$ & $\mathbf{3 . 8 6}(\mathbf{1 . 6 6})$ &. $\mathbf{6 3}(.29)$ &. $\mathbf{1 7}$ & $\mathbf{2 . 2 1}$ & $\mathbf{. 0 2 8}$ & $\mathbf{. 0 7}$ & $\mathbf{1 . 1 9}$ \\
Positive reframing $(\alpha=.69)$ & $5.63(1.70)$ & $-.32(.34)$ & -.09 & -.94 & .349 & -.99 & .35 \\
Use of Emotional support $(\alpha=.65)$ & $4.59(1.65)$ & $.04(.33)$ & .01 & .12 & .909 & -.62 & .69 \\
Use of Instrumental support $(\alpha=.65)$ & $4.71(1.71)$ & $.23(.35)$ & .06 & .65 & .519 & -.46 & .92 \\
Planning $(\alpha=.77)$ & $5.77(1.75)$ & $.59(.38)$ & .17 & 1.55 & .123 & -.16 & 1.34 \\
Humor $(\alpha=.82)$ & $\mathbf{4 . 4 3 ( 2 . 0 0 )}$ & $-.54(.24)$ & -.18 & $-\mathbf{2 . 3 0}$ & $\mathbf{. 0 2 3}$ & $-\mathbf{1 . 0 1}$ &.$- \mathbf{0 8}$ \\
Acceptance $(\alpha=.47)$ & $5.70(1.54)$ & $-.10(.32)$ & -.03 & -.31 & .754 & -.74 & .54 \\
Active Coping $(\alpha=.73)$ & $5.55(1.75)$ & $-.52(.31)$ & -.15 & -1.69 & .093 & -1.13 & .09 \\
Religion $(\alpha=.76)$ & $5.87(1.81)$ & $.07(.25)$ & .02 & .28 & .782 & -.43 & .56 \\
\hline
\end{tabular}

95\% CI. $L B$ Lower Bound, $U B$ Upper Bound; ${ }^{*} p \leq 0.05$; significant values are in bold 
Author Contributions M.O. conceptualized the study. M.O, N.E.Y.D., E.M., \& S.O were all involved in drafting the manuscript. N.E.Y.D. analyzed and interpreted the data. All authors were involved in revising the manuscript and have approved the final version for publication.

Funding This research did not receive any funding from any agency in the public, commercial, or not-for-profit sectors.

Data Availability The dataset generated and/or analyzed during the current study is available from the corresponding author on reasonable request.

\section{Declarations}

Ethical Approval All procedures performed in studies involving human participants were in accordance with the ethical standards of the institutional and/or national research committee and with the 1964 Helsinki declaration and its later amendments or comparable ethical standards.

Informed Consent Informed consent was obtained from all individual participants included in the study.

Declaration of Competing Interests The authors declare no conflict of interest regarding the publication of this paper.

\section{References}

Ahorsu, D. K., Lin, C. Y., Imani, V., Saffari, M., Griffiths, M. D., \& Pakpour, A. H. (2020). The fear of COVID-19 scale: Development and initial validation. International Journal of Mental Health and Addiction. https://doi.org/10.1007/s11469-020-00270-8

Bakioğlu, F., Korkmaz, O., \& Ercan, H. (2020). Fear of COVID-19 and positivity: Mediating role of intolerance of uncertainty, depression, anxiety, and stress. International Journal of Mental Health and Addiction, 1-14. https://doi.org/10.1007/s11469-020-00331-y

Bao, A. M., \& Swaab, D. F. (2019). The human hypothalamus in mood disorders: The HPA axis in the center. IBRO Reports, 6, 45-53. https://doi.org/10.1016/j.ibror.2018.11.008

Bäuerle, A., Teufel, M., Musche, V., Weismüller, B., Kohler, H., Hetkamp, M., ... Skoda, E. M. (2020). Increased generalized anxiety, depression and distress during the COVID-19 pandemic: a cross-sectional study in Germany. Journal of Public Health, 1-7. https://doi.org/10.1093/pubmed/fdaa106

Bischetti, L., Canal, P., \& Bambini, V. (2020). Funny but aversive: A large-scale survey on the emotional response to Covid-19 humor in the Italian population during the lockdown. Lingua, 249, 102963. https://doi.org/10.31234/osf.io/efk93

Cao, W., Fang, Z., Hou, G., Han, M., Xu, X., Dong, J., \& Zheng, J. (2020). The psychological impact of the COVID-19 epidemic on college students in China. Psychiatry Research, 287, 112934. https://doi.org/10.1016/j.psychres.2020.112934

Carver, C. S. (1997). You want to measure coping but your protocol' too long: Consider the brief cope. International Journal of Behavioral Medicine, 4(1), 92-100. https://doi.org/10.1207/ s15327558ijbm0401_6

Carver, C., Scheier, M., \& Weintraub, J. (1989). Assessing coping strategies: A theoretically based approach. Journal of Personality and Social Psychology, 56, 267-283. https://doi.org/10.1037/00223514.56.2.267

Cohen, J. (1992). A power primer. Psychological Bulletin, 112(1), 155159 Retrieved from: http://www2.psych.ubc.ca/ schaller/ 528Readings/Cohen1992.pdf. Accessed 9 Dec 2020
Crawford, S. A., \& Caltabiano, N. J. (2011). Promoting emotional wellbeing through the use of humour. The Journal of Positive Psychology, 6(3), 237-252. https://doi.org/10.1080/17439760. 2011.577087

de Lima, C. V. C., Cândido, E. L., da Silva, J. A., Albuquerque, L. V., de Menezes Soares, L., do Nascimento, M. M., ... Neto, M. L. R. (2020). Effects of quarantine on mental health of populations affected by Covid-19. Journal of Affective Disorders, 275, 253-254. https://doi.org/10.1016/j.jad.2020.06.063.

Dijkstra, M., \& Homan, A. C. (2016). Engaging in rather than disengaging from stress: Effective coping and perceived control. Frontiers in Psychology, 7, 1415. https://doi.org/10.3389/fpsyg. 2016.01415

Eisenbeck, N., Carreno, D. F., \& Uclés-Juárez, R. (2019). From psychological distress to academic procrastination: Exploring the role of psychological inflexibility. Journal of Contextual Behavioral Science, 13, 103-108. https://doi.org/10.1016/j.jcbs.2019.07.007

Faul, F., Erdfelder, E., Lang, A., \& Buchner, A. (2007). G*power 3: A flexible statistical power analysis program for the social, behavioral, and biomedical sciences. Behavior Research Methods, 39(2), 175191. https://doi.org/10.3758/BF03193146

Fofana, N. K., Latif, F., Sarfraz, S., Bashir, M. F., \& Komal, B. (2020). Fear and agony of the pandemic leading to stress and mental illness: An emerging crisis in the novel coronavirus (COVID-19) outbreak. Psychiatry Research, 291, 113230. https://doi.org/10.1016/j. psychres.2020.113230

Folkman, S., \& Lazarus, R. (1980). An analysis of coping in a middle-aged community sample. Journal of Health and Social Behavior, 21(3), 219 239 Retrieved from: http://www.jstor.org/stable/2136617

García, F. E., Barraza-Peña, C. G., Wlodarczyk, A., Alvear-Carrasco, M., \& Reyes-Reyes, A. (2018). Psychometric properties of the briefCOPE for the evaluation of coping strategies in the Chilean population. Psicologia: Reflexão e Crítica, 31(1), 22. https://doi.org/10. 1186/s41155-018-0102-3

Ghana Health Service (2020). COVID-19 Updates. Retrieved from https://www.ghanahealthservice.org/covid19/. Accessed 24 Nov 2020

Hayes, A. F. (2009). Beyond baron and Kenny: Statistical mediation analysis in the new millennium. Communication Monographs, 76(4), 408-420. https://doi.org/10.1080/03637750903310360

Hayes, A. F., \& Rockwood, N. J. (2020). Conditional process analysis: Concepts, computation, and advances in the modeling of the contingencies of mechanisms. American Behavioral Scientist, 64(1), 19-54. https://doi.org/10.1177/F0002764219859633

Holmes, E. A., O'Connor, R. C., Perry, V. H., Tracey, I., Wessely, S., Arseneault, L., ... Ford, T. (2020). Multidisciplinary research priorities for the COVID-19 pandemic: A call for action for mental health science. The Lancet Psychiatry, 7, 547-560. https://doi.org/10.1016/ S2215-0366(20)30168-1.

Jin, Y., Fraustino, J. D., \& Liu, B. F. (2016). The scared, the outraged, and the anxious: How crisis emotions, involvement, and demographics predict publics' conative coping. International Journal of Strategic Communication, 10(4), 289-308. https://doi.org/10.1080/ 1553118X.2016.1160401

Kapasia, N., Paul, P., Roy, A., Saha, J., Zaveri, A., Mallick, R., Barman, B., Das, P., \& Chouhan, P. (2020). Impact of lockdown on learning status of undergraduate and postgraduate students during COVID19 pandemic in West Bengal, India. Children and Youth Services Review, 116, 105194. https://doi.org/10.1016/j.childyouth.2020. 105194

Khalid, I., Khalid, T. J., Qabajah, M. R., Barnard, A. G., \& Qushmaq, I. A. (2016). Healthcare workers emotions, perceived stressors and coping strategies during a MERS-CoV outbreak. Clinical Medicine \& Research, 14(1), 7-14. https://doi.org/10.3121/cmr. 2016.1303 
Kilbane, K. S., Girgla, N., Zhao, L., Barnett, S. L., Berezovsky, A., Lagisetty, K., Lin, J., \& Reddy, R. M. (2020). Adaptive and maladaptive coping mechanisms used by patients with esophageal Cancer after Esophagectomy. Journal of Surgical Research, 258, 1-7. https://doi.org/10.1016/j.jss.2020.07.043

Labrague, L. J., \& De los Santos, J. (2020). Fear of Covid-19, psychological distress, work satisfaction and turnover intention among frontline nurses. Journal of Nursing Management, 29, 395-403. https://doi.org/10.1111/jonm. 13168

Lalande, K. M., \& Bonanno, G. A. (2011). Retrospective memory bias for the frequency of potentially traumatic events: A prospective study. Psychological Trauma Theory Research Practice and Policy, 3(2), 165-170. https://doi.org/10.1037/a0020847

Lazarus, R. S. (1999). Hope: An emotion and a vital coping resource against despair. Social Research, 66(2), 653-678 Retrieved from: http://www.jstor.org/stable/40971343. Accessed 9 Dec 2020

Lee, H., Yoon, J. Y., Kim, I., \& Jeong, Y. H. (2013). The effects of personal resources and coping strategies on depression and anxiety in patients with chronic obstructive pulmonary disease. Heart \& Lung, 42(6), 473-479. https://doi.org/10.1016/j.hrtlng.2013.07.009

Li, J., Yang, Z., Qiu, H., Wang, Y., Jian, L., Ji, J., \& Li, K. (2020). Anxiety and depression among general population in China at the peak of the COVID-19 epidemic. World Psychiatry, 19(2), 249250.

Lovibond, P. F., \& Lovibond, S. H. (1995). The structure of negative emotional states: Comparison of the depression anxiety stress scales (DASS) with the Beck depression and anxiety inventories. Behaviour Research and Therapy, 33(3), 335-343.

Maxwell, W. (2003). The use of gallows humor and dark humor during crisis situation. International Journal of Emergency Mental Health, 5(2), 93-98.

Mbiti, J. S. (1969). African religions \& philosophy. Heinemann.

Miller, B. L. (2020). Science denial and COVID conspiracy Theories: Potential Neurological Mechanisms and Possible Responses. JAMA, 324(22), 2255-2256. https://doi.org/10.1001/jama.2020.21332

Oplatka, I., \& Iglan, D. (2020). The emotion of fear among schoolteachers: Sources and coping strategies. Educational Studies, 46(1), 92-105. https://doi.org/10.1080/03055698.2018.1536876

Park, S. H., Song, Y. J. C., Demetriou, E. A., Pepper, K. L., Thomas, E. E., Hickie, I. B., \& Guastella, A. J. (2020). Validation of the 21-item depression, anxiety, and stress scales (DASS-21) in individuals with autism spectrum disorder. Psychiatry Research, 291, 113300. https://doi.org/10.1016/j.psychres.2020

Preacher, K. J., \& Hayes, A. F. (2008). Asymptotic and resampling strategies for assessing and comparing indirect effects in multiple mediator models. Behavior Research Methods, 40(3), 879-891. https:// doi.org/10.3758/BRM.40.3.879

Rettie, H., \& Daniels, J. (2021). Coping and tolerance of uncertainty: Predictors and mediators of mental health during the COVID-19 pandemic. American Psychologist, 76(3), 427-437. https://doi.org/ 10.1037/amp0000710

Rodríguez-Rey, R., Garrido-Hernansaiz, H., \& Collado, S. (2020). Psychological impact and associated factors during the initial stage of the coronavirus (COVID-19) pandemic among the general population in Spain. Frontiers in Psychology, 11, 1540. https://doi.org/ 10.3389/fpsyg.2020.01540

Romer, D., \& Jamieson, K. H. (2020). Conspiracy theories as barriers to controlling the spread of COVID-19 in the US. Social Science \& Medicine, 263, 113356. https://doi.org/10.1016/j.socscimed.2020. 113356

Satici, B., Saricali, M., Satici, S. A., \& Griffiths, M. D. (2020). Intolerance of uncertainty and mental well-being: Serial mediation by rumination and fear of COVID-19. International Journal of Mental Health and Addiction, 1-12. Advance online publication. https://doi.org/10.1007/s11469-020-00305-0
Serafini, G., Parmigiani, B., Amerio, A., Aguglia, A., Sher, L., \& Amore, M. (2020). The psychological impact of COVID-19 on the mental health in the general population. QJM: An International Journal of Medicine, 113(8), 531-537. https://doi.org/10.1093/qjmed/hcaa201

Shaukat, N., Ali, D. M., \& Razzak, J. (2020). Physical and mental health impacts of COVID-19 on healthcare workers: A scoping review. International Journal of Emergency Medicine, 13(1), 1-8. https:// doi.org/10.1186/s12245-020-00299-5

Siebenhaar, K. U., Köther, A. K., \& Alpers, G. W. (2020). Dealing with the COVID-19 infodemic: Distress by information, information avoidance, and compliance with preventive measures. Frontiers in Psychology, 11, 2981. https://doi.org/10.3389/fpsyg.2020.567905

Skeffington, P. M., Rees, C. S., \& Mazzucchelli, T. (2017). Trauma exposure and post-traumatic stress disorder within fire and emergency services in Western Australia. Australian Journal of Psychology, 69(1), 20-28. https://doi.org/10.1111/ajpy.12120

Sullivan, H. (2020, May 13). Why should you cry? Ghana's dancing pallbearers find new fame during Covid-19. The Guardian. Retrieved from https://www.theguardian.com/world/2020/may/14/ why-should-you-cry-ghanas-dancing-pallbearers-find-new-fameduring-covid-19. Accessed 10 Dec 2020

Taha, S., Matheson, K., Cronin, T., \& Anisman, H. (2014). Intolerance of uncertainty, appraisals, coping, and anxiety: The case of the $2009 \mathrm{H}$ $1 \mathrm{~N} 1$ pandemic. British Journal of Health Psychology, 19(3), 592605. https://doi.org/10.1111/bjhp.12058

Thompson, S. C., \& Ting, S. A. (2012). Avoidance denial versus optimistic denial in reaction to the threat of future cardiovascular disease. Health Education \& Behavior, 39(5), 620-629. https://doi.org/ 10.1177/1090198111428154

Umucu, E., \& Lee, B. (2020). Examining the impact of COVID-19 on stress and coping strategies in individuals with disabilities and chronic conditions. Rehabilitation Psychology, 65(3), 193-198. https://doi.org/10.1037/rep0000328193

Wang, H., Xia, Q., Xiong, Z., Li, Z., Xiang, W., Yuan, Y., Liu, Y., \& Li, Z. (2020). The psychological distress and coping styles in the early stages of the 2019 coronavirus disease (COVID-19) epidemic in the general mainland Chinese population: A web-based survey. PLoS One, 15(5), e0233410. https://doi.org/10.1371/journal.pone. 0233410

WHO (2020a). Naming the coronavirus disease (COVID-19) and the virus that causes it. Retrieved from https://www.who.int/ emergencies/diseases/novel-coronavirus-2019/technical-guidance/ naming-the-coronavirus-disease-(covid-2019)-and-the-virus-thatcauses-it. Accessed 15 Dec 2020

WHO (2020b). WHO Coronavirus Disease (COVID-19) Dashboard. Retrieved from https://covid19.who.int/. Accessed 15 Dec 2020

Wnuk, A., Oleksy, T., \& Maison, D. (2020). The acceptance of Covid-19 tracking technologies: The role of perceived threat, lack of control, and ideological beliefs. PLoS One, 15(9), e0238973. https://doi.org/ 10.1371/journal.pone.0238973

Ye, Z., Yang, X., Zeng, C., Wang, Y., Shen, Z., Li, X., \& Lin, D. (2020). Resilience, social support, and coping as mediators between COVID-19-related stressful experiences and acute stress disorder among college students in China. Applied Psychology. Health and Well-Being, 12, 1074-1094. https://doi.org/10.1111/aphw.12211

Zanon, C., Brenner, R. E., Baptista, M. N., Vogel, D. L., Rubin, M., AlDarmaki, F. R.,... \& Zlati, A. (2021). Examining the dimensionality, reliability, and invariance of the depression, anxiety, and stress scale-21 (DASS-21) across eight countries. Assessment, 28(6), 1531-1544. https://doi.org/10.1177/1073191119887449

Publisher's Note Springer Nature remains neutral with regard to jurisdictional claims in published maps and institutional affiliations. 